\title{
Analysis of polymorphisms Leiden Factor V G1691A and prothrombin G20210A as risk factors for acute myocardial infarction
}

\author{
Giusi Irma Forte $\cdot$ Loredana Vaccarino $\cdot$ Marisa Palmeri • \\ Angelo Branzi • Claudio M. Caldarera $\cdot$ Letizia Scola • \\ Calogero Caruso • Federico Licastro • Domenico Lio
}

Received: 27 January 2011 / Accepted: 23 February 2011/Published online: 15 September 2011

(C) Springer Science+Business Media B.V. 2011

\begin{abstract}
Thrombotic risk increases in elderly, therefore, the understanding of the genetic predisposition of hypercoagulability could make the difference in the prevention of venous and/or arterial thrombotic events. Laboratory evaluation of hyperfibrinogenemia, increased Factor VII levels, antiphospholipid antibodies presence and hyperhomocysteinemia are considered to have a consistent high predictivity for arterial thrombophilic diseases. Anyway, a large debate exists on the validity of testing Leiden Factor V (FV) G1691A and/or prothrombin (FII) G20210A polymorphisms in patients affected by arterial thrombotic diseases, despite of the several observations described. Here we report data strongly suggesting that at least the FII G20210A polymorphism might be
\end{abstract}

G. I. Forte $(\varangle) \cdot$ L. Vaccarino - M. Palmeri .

L. Scola · C. Caruso · D. Lio

Department of Biopathology and Medical and Forensic Biotechnology, University of Palermo, Corso Tukory 211, 90134 Palermo, Italy

e-mail: giusiforte@unipa.it

\author{
A. Branzi \\ Cardiovascular Department, University of Bologna, \\ Bologna, Italy \\ C. M. Caldarera \\ Department of Biochemistry, University of Bologna, \\ Bologna, Italy \\ F. Licastro \\ Department of Sperimental Pathology, University \\ of Bologna, Bologna, Italy
}

considered an important risk factor for acute myocardial infarction in aged patients (55-80 years old). On the other hand, in spite of a not different genotypic and allelic distribution for the Leiden FV G1691A mutation, the presence of one or both the two polymorphisms is significantly higher among cases than in controls. In conclusion, our data suggest that FII G20210A and/or Leiden FV might be involved as risk factor for arterial disorders in about 5\% of old subjects, justifying the opportunity of a genetic screening and an eventual preventive treatment, in particular in old subjects in which other and major risk factors, as hypertension and atherosclerosis, are detected.

Keywords Leiden Factor V · Prothrombin · Stroke · Guidelines · Arterial thrombosis

\section{Introduction}

It is well known that thrombotic risk increases in elderly, as physiological aging is associated with increased plasma levels of many blood coagulation proteins, with alterations of platelets and fibrinolysis impairment (Mari et al. 2008), so that pathologies as atherosclerosis, cardiovascular disease or stroke are typically considered aging associated diseases.

Therefore, the understanding of the genetic predisposition of hypercoagulability could make the difference in the prevention of venous and/or arterial 
thrombotic events, with high benefits in health costs. According to this view, several scientific societies involved in this field have elaborated guidelines suggesting a list of recommended tests for the screening of congenital or acquired thrombophilic diseases (De Stefano et al. 1996; Lane et al. 1996) in categories of risk patients. In addition, in different studies mutations of genes not involved in clot formation and regulation have been found to play a crucial role in the susceptibility for arterial thrombotic disorders (Lio et al. 2004; Listì et al. 2008; Billeci et al. 2009), but generally they're not retained useful in public health programs for the risk's evaluation profile, due to their modest contribution to the benefits/costs balance.

Actually, in order to test the congenital or acquired predisposition to the deep venous thrombosis, mutations occurring on genes coding for antithrombin III (AT), protein C (PC), protein S (PS), Leiden Factor V (FV) and prothrombin (FII) G20210A, or their deficiency, plus dysfibrinogenemia, hyperhomocysteinemia or antiphospholipid antibodies presence, are currently searched in patients undergoing surgery, pregnant or using oral contraceptives women, patients with family history, previous episodes or youth's events (Testa et al. 2004; Tripodi and Mannucci 2001).

Whereas, as it is largely recognized, only hyperfibrinogenemia, increased Factor VII (FVII) levels, antiphospholipid antibodies presence and hyperhomocysteinemia are considered to have a consistent high predictivity for the susceptibility to arterial thrombophilic diseases (Van Cott et al. 2002; Kahn 2003).

However, although several studies have observed an association of Leiden FV and FII G20210A with arterial thrombotic disorders, especially in the association with the juvenile cases (Kim and Becker 2003), and it has been reported that these tests can be considered useful in certain situations, such as in the evaluation of the risk profile of young patients and/or in their relatives (Soo and Davidson 2007), there is no consistent evidence for the recommendation of routine typing for Leiden FV, FII, AT III, PC and PS in patients having risk of stroke or arterial thrombotic disease (Ridker et al. 1995; Tripodi and Mannucci 2001; Ridker et al. 1999).

Moreover, a strong coagulatory potential increasing characterizes elderly, so that age itself plays an important role as risk factor in the onset of thromboembolic diseases, added to other risk factors as smoking, hypertension or diabetes, anyway, the contribution of genetic polymorphisms and in particular of Leiden FV and FII G20210A to the susceptibility to arterial thrombotic events in old people remain to be demonstrated.

Here, we report data on the evaluation of the frequencies of polymorphisms FV G1691A and FII G20210A in a group of 164 patients aged between 55 and 80 years old, affected by acute myocardial infarction (AMI), to give an insight on the opportunity to execute this genetic tests on restricted category of risk's patients in advanced age, in order to provide an instrument to clinicians to act in the prevention of the thrombotic event.

\section{Materials and methods}

Subjects

In this study, we analyzed a cohort of patients affected by acute myocardial infarction and one other of unrelated age matched controls. The cohort comprised 164 patients aged between 55-80 years old with a mean age of 67 years, of which $80 \%$ were male, affected by acute myocardial infarction, who were diagnosed at the cardiac unit of Bologna University Hospital. The diagnosis of acute myocardial infarction was based on typical electrocardiographic changes and standard laboratory findings, in particular troponin $I$ value greater than a decision limit of $3.0 \mathrm{ng} / \mathrm{ml}$, (Peetz et al. 2006), confirmed by echocardiography and coronary angiography. 253 controls matched for age, of which about $75 \%$ were male from the same geographic origin, who had no clinical history of age related diseases were also recruited. Informed consent to recruitment in the study was obtained from each subject according to European guidelines and Italian laws.

G1691A of FV and 20210GA of FII Allele

Discrimination assay

The blood specimens were collected in EDTA sterile tubes, and stored in freezer at $-20^{\circ} \mathrm{C}$. Then, DNA was extracted using a salting out protocol (Miller et al. 1988), resuspended in Tris-EDTA buffer and stored at $-20^{\circ} \mathrm{C}$. Typing of FV mutation was performed for 214 out of 253 control' and 149 out of 164 patient' DNA samples, whereas 222 out of 253 controls and 150 out 
of 164 patients were typed for FII polymorphism. Among these, 207 out of 253 controls and 148 patients out of 164 were typed for both the two gene variants.

Two dedicated and pre-made Taqman Assays developed by Applied Biosystem (USA) were used to perform the Allelic Discrimination tests for the typing of the polymorphisms FV G1691A and FII G20210A.

Briefly, 10 ng of DNA for each sample were used in a PCR reaction, containing $1 \times$ optimized master mix and $1 \times$ specific primers/probes mix assay, according to manufactory protocol in a final volume of $25 \mu \mathrm{l}$.

For each polymorphism two identical probes, except for the central nucleotide that specifically recognizes the single nucleotide polymorphism (SNP) were used, each one labeled at the $5^{\prime}$ extremity with different dyes, FAM and VIC in this case, and at the $3^{\prime}$ extremity with a quencher dye, that in this case was the Minor Groove Binder (MGB) dye.

Then, the amplification was performed in 7300 RealTime ABI Prism PCR System (Applied Biosystems, USA), using a standard amplification protocol (1 cycle of $2^{\prime}$ at $50^{\circ} \mathrm{C} ; 1$ cycle of $10^{\prime}$ at $95^{\circ} \mathrm{C}$ and 40 cycles of $15^{\prime \prime}$ at $95^{\circ} \mathrm{C}$ plus $15^{\prime \prime}$ at $60^{\circ} \mathrm{C}$ ), and the results available in the report sheet of 7300 System SDS v1.3 Software.

Moreover, samples were graphically grouped in three genotypic clusters, easily recognizable in the Allelic Discrimination plot on the basis of the two probe's fluorescence intensity emissions, whereas the uncertain cases were also evaluated for the grow up of the fluorescence emission curve of each dyes on the component's sheet.

Statistical analysis

The data were tested for goodness of fit between the observed and expected genotype $(3 \times 2$ tables $)$ and allele $(2 \times 2$ tables $)$ frequencies. Fisher's exact tests were performed to calculate significant different genotype or allelic distributions between patients with acute myocardial infarction and age matched controls. Odds ratio and $95 \%$ of confidence intervals were calculated with Woolf's approximation to quantify the risk in carriers of minor allelic variants. Mantel-Haenszel procedure for the trend analysis was applied to evaluate the effect of gene polymorphism combinations.

\section{Results}

Table 1 shows the analysis of genotypic and allelic frequencies of the single nucleotide polymorphism located at the position G20210A of the prothrombin (FII) $3^{\prime}$ UTR region among the 150 patients and 222 healthy controls. Both patient' and control' genetic frequencies fit the Hardy-Weinberg equilibrium. We observed a considerable significant increase of the minor allele $(\mathrm{A})(P=0.0031)$, that is represented with a percentage of $5.3 \%$ among patients, against $1.35 \%$ among the healthy controls. Accordingly, a significant different genotypic distribution characterized by a consistent increase of the A positive subjects (10.0 vs. $2.7 \%)(P=0.0048$, Odds ratio $4.00 ; 95 \%$ Confidence Interval 1.51-10.56) is also observable.

Instead, genotypic and allelic distribution were not different for the polymorphism Leiden FV G1691A between patients and controls, $(P>0.05)$, as the allele frequency in patients was $2.7 \%$, overlapping that one observable for the control group (2.1\%) (Table 2).

On the other hand, it is well known that the protection or susceptibility effect of a single polymorphism might be modulated by both an additive/synergic or antagonistic pressure through the presence of other polymorphisms in genes involved in the same

Table 1 FII 20210G $\rightarrow$ A. Genotypic and allelic frequencies of polymorphism G20210A of prothrombin gene observed in 150 over-fifty-five acute myocardial infarction affected patients and healthy age matched controls

\begin{tabular}{|c|c|c|c|c|c|c|}
\hline & \multirow[t]{2}{*}{$N$} & \multicolumn{3}{|c|}{ Genotype frequencies } & \multicolumn{2}{|c|}{ Allele frequencies } \\
\hline & & $\mathrm{G} / \mathrm{G}$ & $\mathrm{G} / \mathrm{A} * *$ & $\mathrm{~A} / \mathrm{A} * *$ & $\mathrm{G}$ & $A^{*}$ \\
\hline Cases & 150 & $90 \%(135)$ & $9.3 \%(14)$ & $0.7 \%$ & $94.7 \%(284)$ & $5.3 \%(16)$ \\
\hline Controls & 222 & $97.3 \%(216)$ & $2.7 \%(6)$ & $0 \%(0)$ & $98.65 \%(438)$ & $1.35 \%(6)$ \\
\hline
\end{tabular}

*20210A allele frequency was found significantly increased in the patients group vs. controls.** Accordingly, a significant increase of the percentage of acute myocardial infarction patients carrying A positive genotypes was observed $(P=0.0048$, Odds ratio 4.00; 95\% Confidence Interval 1.51-10.56) 
Table 2 FV 1691G $\rightarrow$ A. Genotypic and allelic frequencies of polymorphism G1691A of Factor V gene observed in 150 over- fiftyfive acute myocardial infarction affected patients and healthy age matched controls

\begin{tabular}{|c|c|c|c|c|c|c|}
\hline & \multirow[t]{2}{*}{$N$} & \multicolumn{3}{|c|}{ Genotype frequencies } & \multicolumn{2}{|c|}{ Allele frequencies } \\
\hline & & $\mathrm{G} / \mathrm{G}$ & $\mathrm{G} / \mathrm{A}$ & $\mathrm{A} / \mathrm{A}$ & G & A \\
\hline Cases & 149 & $94.6 \%(141)$ & $5.4 \%(8)$ & $0 \%(0)$ & $97.3 \%(290)$ & $2.7 \%(8)$ \\
\hline Controls & 214 & $95.8 \%(205)$ & $4.2 \%(9)$ & $0 \%(0)$ & $97.9 \%(419)$ & $2.1 \%(9)$ \\
\hline
\end{tabular}

Genotypic and allelic distribution were not different between patient and control groups

metabolic or functional pathway. In this view, we tested the hypothesis that the presence of both the two mutations could increase the risk of susceptibility or onset of cardiovascular events. In particular, MantelHaenszel procedure allows to find a significant trend for the increase of single and double locus heterozygosis in patients affected by acute myocardial infarction $(P=0.013)$, with a cumulative odds ratio of 2.79 (95\% Confidence Interval 1.28 to 6.05) (Table 3). Actually, among 148 patients that it was possible to test, 5 patients carrying both the two mutations in heterozygosis were found. Furthermore, also the presence of at least one of the two polymorphisms in heterozygosis (FV G1691A or FII G20210A) is prevalent among cases $(7.3 \%)$ than in controls $(6.3 \%)$. The calculated odd ratio for the presence of at least one mutation is 1.82 (95\% C.I. 0.8483 to 3.916), while in the presence of double heterozygous condition the odd ratio became 16.018 (95\% C.I. 0.8782 to 292.16 ).

\section{Discussion}

Susceptibility to the onset of arterial disorders are surely conditioned by the presence of clinical/environmental risk factors, such as smoking, advanced age, elevated cholesterol/LDL plasma levels, hypertension, diabetes, that strongly predispose to arterial thrombotic events.
A large debate exists on the risk association among Leiden Factor V (FV) G1691A and/or prothrombin (FII) G20210A polymorphisms and arterial disorders, despite of the several observations reported in a consistent collection of literature's papers showing data regarding different cohorts of patients affected by arterial thrombotic diseases, having different age ranges and geographical proveniences (Mazoyer et al. 2009; Kahn 2003; Ridker et al. 1995; Ozmen et al. 2009; Ridker et al. 1999; Kim and Becker 2003; Soare and Popa 2010).

Nevertheless, it has been supposed that there are not enough evidence for the suggestion of diagnostic investigation of these mutations, whereas guidelines only suggest the execution of other tests that are considered to have a major predictive value, such as hyperfibrinogenemia, increased Factor VII levels, antiphospholipid antibodies presence and hyperhomocysteinemia (Soo and Davidson 2007, Tripodi and Mannucci 2001).

Significant association among Leiden FV and FII G20210A and stroke in young $<50$ years old patients have been described (Kim and Becker 2003; Gemmati et al. 2001; Soare and Popa 2010). So these mutations could make the difference for the susceptibility to stroke in patients in which there are not yet compliances generated by age-related defects and diseases and may justify the evaluation of these genetic factors both in patients and their relatives. This is at least

Table 3 FV G1691A and FII G20210A combinations. Analysis of genotypic combinations of polymorphisms FV G1691A and FII G20210A observed in 148 over- fifty-five acute myocardial infarction affected patients and healthy age matched controls

\begin{tabular}{lllllll}
\hline \multicolumn{1}{c}{$N$} & Genotypic frequencies & & \\
\cline { 3 - 6 } & & $\begin{array}{l}\text { FVG1691 and FIIG20210 } \\
\text { homozygous wild type }\end{array}$ & $\begin{array}{l}\text { Single locus } \\
\text { heterozygosis* }\end{array}$ & $\begin{array}{l}\text { Double locus } \\
\text { heterozygosis* }\end{array}$ & $\begin{array}{l}\text { Single locus minor } \\
\text { allele homozygosis }\end{array}$ & $\begin{array}{l}\text { Double locus minor } \\
\text { allele homozygosis }\end{array}$ \\
\hline Cases & 148 & $88.5 \%(131)$ & $7.4 \%(11)$ & $3.4 \%(5)$ & $0.7 \%(1)$ & $0 \%(0)$ \\
Controls & 207 & $93.5 \%(194)$ & $6.5 \%(13)$ & $0 \%(0)$ & $0 \%(0)$ & $0 \%(0)$ \\
\hline
\end{tabular}

*Mantel-Haenszel procedure allows to find a significant trend for the increase of single and double locus heterozygosis in patients affected by acute myocardial infarction $(P=0.0113)$, the cumulative odd ratio is 2.79 (95\% Confidence Interval 1.28 to 6.05 ) 
reputed licit by some authors (Soo and Davidson 2007).

As well known, hypercoagulability, remains an important cause of haemostatic unbalance, growing with age advancing (Mari et al. 2008), but data on the impact of these two mutations on arterial disorders risk on selected cohort of aged patients are not exhaustive. Here we report data strongly suggesting that at least the FII G20210A polymorphism might be considered a risk factor for acute myocardial infarction (AMI) in aged patients (55-80 years old). Actually, we observed a significant increase of 20210A FII allele associated to the reported higher plasma prothrombin level respect to the wild type ones (Mazoyer et al. 2009).

The presence of G 20210 A substitution in the $3^{\prime}$ upstream untranslated region of prothrombin gene seems to play a regulatory role in gene expression (Poort et al. 1996). Actually, the $\mathrm{G}$ to A transition at nucleotide position 20,210, at or near the cleavage site of the mRNA precursor, to which poly A is added, seems to stabilize mRNA allowing an increased production of the corresponding protein (Poort et al. 1996).

The biological plausibility that a higher prothrombin level related to the 20210A variant may also confer an increased risk of arterial disease seems well supported by studies in which an excessive thrombin generation has been described in individuals at high risk of fatal coronary artery disease (Miller et al. 1996). To date, however, studies attempting to answer this question have yielded conflicting results. In some reports, being a carrier of the mutation was associated with an increased risk of AMI (Franco et al. 1999; Miller et al. 1996). Other studies failed to establish any association between the 20210A allele and AMI. (Russo et al. 2001; Ridker et al. 1999).

On the other hand, as above described, in spite of a similar genotypic and allelic distribution observed for the Leiden FV G1691A mutation, the percentage of patients bearing one or both the two polymorphisms is more or significantly increased respect to the controls.

As it is known, an adenine instead of a guanine replacement in the position 1,691 of FV gene introduces a critical aminoacid change in the protein (Arg506Gln), that make the FV resistant to the attack of PC, producing an increasing of hypercoagulability (De Stefano and Leone 1995).

As reported by Gemmati et al. (2001), in a family clinical study it was observed that a patient, who exhibited combined heterozygosis for FV G1691A and FII G20210A mutations, was affected by spontaneous and early clinical onset recurrences of deep-vein thrombosis and pulmonary embolism and similar data was described among the relatives (Gemmati et al. 2001). This case report seems to suggest that the synergy of inherited factors and transient risk conditions could play a key role in the occurrence of thrombotic accidents.

Our results seem to suggest that individuals carriers for double combined heterozygous FV G1691A and FII G20210A mutations may be particularly susceptible to AMI.

The possibility that this selection could be related to the age range of individuals analyzed should be taken in account. Beside, we matched for age our group of controls. Moreover, in previous studies we did not observe differences in genotype and allele frequencies in populations stratified for age, as centenarians ( $>90$ years old) had similar frequencies respect to young controls (Forte et al. 2009). Accordingly, no age stratification difference was observed in a study involving 6,154 French individuals. (Mazoyer et al. 2009) .

In conclusion, our data seem to suggest that prothrombin G20210A variant alone or in combination with Leiden FV might be involved as risk factor for AMI in about $5 \%$ of subjects $>55$ years old.

However, as above reported, previous studies have shown negative associations among cardiovascular events and prothrombin G20210A and/or Leiden FV polymorphisms. In this view, the relatively small number of subjects typed for the two polymorphisms in our study might affect the strength of the statistical comparison. So, our data necessitate to be confirmed in a larger population of aged people. This should be taken in account before to draw definitive conclusions on the opportunity of a genetic screening and an eventual preventive treatment, in particular in old subject population in which other and major risk factors as hypertension and atherosclerosis are commonly detected.

Acknowledgments This research was supported by grants from the Ministry of Education, University and Research (Cofin. to D.L. and local initiatives to D.L.), and from Ministry of Health. G.I.F. is a Phd researcher, working on a project funded by a grant received by Italian Ministry of Education, University and Research, and this work represents an implementation of her own final report. 


\section{References}

Billeci AM, Agnelli G, Caso V (2009) Stroke pharmacogenomics. Expert Opin Pharmacother 10(18):2947-2957

De Stefano V, Leone G (1995) Resistance to activated protein C due to mutated factor $\mathrm{V}$ as a novel cause of inherited thrombophilia. Haematologica 80(4):344-356

De Stefano V, Finazzi G, Mannucci PM (1996) Inherited thrombophilia: pathogenesis, clinical syndromes, and management. Blood 1 87(9):3531-3544

Forte GI, Scola L, Vaccarino L, Sanacore M, Palmeri M, La Piana S, Pipitone M, Grimaudo S, Caruso C (2009) Analysis of FV-Leiden and Facor II G20210A SNP frequencies in a group of Sicilian nonagenarians. J Nutr Health Aging 13(1):S378

Franco RF, Trip MD, ten Cate H, van den Ende A, Prins MH, Kastelein JJ, Reitsma PH (1999) The $20210 \mathrm{G} \rightarrow \mathrm{A}$ mutation in the $3^{\prime}$ untranslated region of the prothrombin gene and the risk for arterial thrombotic disease. $\mathrm{Br} \mathrm{J}$ Haematol 104:50-54

Gemmati D, Serino ML, Moratelli S, Tognazzo S, Ongaro A, Scapoli GL (2001) Coexistence of factor V G1691A and factor II G20210A gene mutations in a thrombotic family is associated with recurrence and early onset of venous thrombosis. Haemostasis 31(2):99-105

Kahn M (2003) Hypercoagulability as a cause of stroke in adults. South Med J 96:350-353

Kim RJ, Becker RC (2003) Association between factor V Leiden, prothrombin G20210A, and methylenetetrahydrofolate reductase C677T mutations and events of the arterial circulatory system: a meta-analysis of published studies. Am Heart J 146(6):948-957

Lane DA, Mannucci PM, Bauer KA, Bertina RM, Bochkov NP, Boulyjenkov V, Chandy M, Dahlbäck B, Ginter EK, Miletich JP, Rosendaal FR, Seligsohn U (1996) Inherited thrombophilia. Thromb Haemost 76(5):651-662

Lio D, Candore G, Crivello A, Scola L, Colonna-Romano G, Cavallone L, Hoffmann E, Caruso M, Licastro F, Caldarera CM, Branzi A, Franceschi C, Caruso C (2004) Opposite effects of interleukin 10 common gene polymorphisms in cardiovascular diseases and in successful ageing: genetic background of male centenarians is protective against coronary heart disease. J Med Genet 41(10):790-794

Listì F, Caruso M, Incalcaterra E, Hoffmann E, Caimi G, Balistreri CR, Vasto S, Scafidi V, Caruso C, Candore G (2008) Pro-inflammatory gene variants in myocardial infarction and longevity: implications for pharmacogenomics. Curr Pharm Des 14(26):2678-2685

Mari D, Coppola R, Provenzano R (2008) Hemostasis factors and aging. Exp Gerontol 43(2):66-73

Mazoyer E, Ripoll L, Gueguen R, Tiret L, Collet JP, dit Sollier CB, Roussi J, Drouet L (2009) Prevalence of factor V Leiden and prothrombin G20210A mutation in a large
French population selected for nonthrombotic history: geographical and age distribution. Blood Coagul Fibrinolysis 20(7):503-510

Miller SA, Dykes DD, Polesky HF (1988) A simple salting out procedure for extracting DNA from human nucleated cells. Nucl Acid Res 16:1215-1220

Miller GJ, Bauer KA, Barzegar S, Cooper JA, Rosenberg RD (1996) Increased activation of the hemostatic system in men at high risk of fatal coronary heart disease. Thromb Haemost 75:767-771

Ozmen F, Ozmen MM, Ozalp N, Akar N (2009) The prevalence of factor V (G1691A), MTHFR (C677T) and PT (G20210A) gene mutations in arterial thrombosis. Ulus Travma Acil Cerrahi Derg 15(2):113-119

Peetz D, Schweigert R, Jachmann N, Post F, Schinzel H, Lackner KJ (2006) Method comparison of cardiac marker assays on PATHFAST, StratusCS, AxSYM, Immulite 2000, triage, elecsys and cardiac reader. Clin Lab 52(11-12): 605-614

Poort SR, Rosendaal FR, Reitsma PH, Bertina RM (1996) A common genetic variation in the $3^{\prime}$-untranslated region of the prothrombin gene is associated with elevated plasma prothrombin levels and an increase in venous thrombosis. Blood 88:3698-3703

Ridker PM, Hennekens CH, Lindpaintner K, Stampfer MJ, Eisenberg PR, Miletich JP (1995) Mutation in the gene coding for coagulation factor $\mathrm{V}$ and the risk of myocardial infarction, stroke, and venous thrombosis in apparently healthy men. N Engl J Med 332(14):912-917

Ridker PM, Hennekens CH, Miletich JP (1999) G20210A mutation in prothrombin gene and risk of myocardial infarction, stroke, and venous thrombosis in a large cohort of US men. Circulation 99(8):999-1004

Russo C, Girelli D, Olivieri O, Guarini P, Manzato F, Pizzolo F, Zaia B, Mazzucco A, Corrocher R (2001) G20210A prothrombin gene polymorphism and prothrombin activity in subjects with or without angiographically documented coronary artery disease. Circulation 103:2436-2440

Soare AM, Popa C (2010) Deficiencies of proteins C, S and antithrombin and factor $\mathrm{V}$ Leiden and the risk of ischemic strokes. J Med Life 3(3):235-238

Testa S, Antonucci G, Intra E, Martini G, Pedrini S, Alatri A, Bader R, Manzato F (2004) Gli screening per trombofilia. Riv Med Lab_JLM 5(2):118

Tripodi A, Mannucci PM (2001) Laboratory investigation of thrombophilia. Clin Chem 47(9):1597-1606

Van Cott E, Laposata M, Prins MH (2002) Laboratory evaluation of hypercoagulability with venous or arterial thrombosis. Arch Pathol Lab Med 126:1281-1295

Soo L, Davidson P (2007) Laboratory testing for thrombophilia in arterial thrombosis. QML Pathology Medical PUB/MR/ 545 , version 2 